\title{
Etude acoustique de la production de glides dans la maladie de Parkinson
}

\author{
Virginie Roland ${ }^{1 *}$, Véronique Delvaux ${ }^{1,2}$, Kathy Huet $^{1}$, Myriam Piccaluga ${ }^{1}$ et Bernard \\ Harmegnies $^{1}$ \\ ${ }^{1}$ Service de Métrologie et Sciences du Langage, Université de Mons, 7000 MONS, Belgique \\ ${ }^{2}$ Fonds de la Recherche Scientifique, Belgique
}

\begin{abstract}
Résumé. Dans cet article, les résultats d'une étude acoustique sur la production de glides par des locuteurs atteints de la maladie de Parkinson et des locuteurs témoins sont présentés. Notre objectif est d'explorer la dynamique des articulateurs supralaryngés dans la MP, en particulier chez des locuteurs MP non dysarthriques. Nos résultats montrent des différences entre les deux groupes de sujets (locuteurs MP et témoins), en particulier en ce qui concerne l'amplitude des mouvements et la localisation dans le plan F1-F2. Nous notons également que ces différences se produisent en l'absence de dysarthrie, suggérant l'identification de phénomènes infracliniques dans la maladie de Parkinson.
\end{abstract}

\begin{abstract}
Acoustic study of glides production in Parkinson's disease. In this paper, results of an acoustic study of glides produced by both patients suffering from Parkinson's disease and control subjects are presented. Our aim is to explore the dynamics of supra-laryngeal articulators in $\mathrm{PD}$, in particular in non dysarthric parkinsonian. Results show differences between the two groups (PD and control speakers), in particular as regards movement amplitude and location in the F1-F2 plane. We also note that these differences take place in the absence of dysarthria, suggesting the identification of infraclinic phenomena in Parkinson's disease.
\end{abstract}

\section{Introduction}

Nos travaux sur la production de la parole dans la maladie de Parkinson (MP) s'inscrivent dans un espace linguistique spécifique : celui de la langue française. En effet, comme nous l'exposerons par la suite, même si bon nombre de travaux portant sur les caractéristiques de la parole de personnes atteintes de la MP ont émergé depuis maintenant plusieurs décennies, force est de constater que fort peu se centrent sur des personnes ayant le français pour langue maternelle et/ou sur des productions verbales en langue française. Le français se différencie pourtant de l'anglais (langue étudiée dans la plupart des recherches en la matière) à divers égards, et il est raisonnable de penser que l'observation du traitement, par les personnes atteintes de la MP, de certains des objets phoniques qu'il comporte, est de

*Corresponding author : virginie.roland@umons.ac.be 
nature à mieux caractériser les difficultés que la maladie impose aux locuteurs. Afin d'apporter une contribution au comblement de cette lacune, c'est auprès de participants s'exprimant usuellement en français que nous recueillerons nos observations.

La deuxième particularité de la présente contribution réside dans la spécificité des sons de parole sur lesquels elle se centre. Ce choix résulte d'un constat : les effets majeurs de la MP concernent le contrôle du mouvement. Or, les recherches classiquement centrées sur la parole des personnes atteintes de la MP se basent, dans leur majorité, sur l'analyse de phénomènes sonores à cible statique et, de ce fait, produits par un conduit vocal nécessairement immobilisé. Les voyelles monophtongues, en particulier, ont fait l'objet d'une attention particulière. Ces sons ne sont certes pas dépourvus d'intérêt puisqu'ils requièrent l'association entre la commande laryngée et le contrôle des articulateurs supraglottiques. La nature des phénomènes sonores impliqués est telle, par ailleurs, que le recours, pour leur étude, à des investigations à base spectrale est pleinement justifiée, ce qui permet d'aisément convoquer les méthodes d'analyse concernant la parole ordinaire. Il n'en reste pas moins qu'en se cantonnant à l'observation de ces sons, on restreint le champ de regard à des phases de locution articulatoirement caractérisées par l'immobilité. Ce raisonnement a conduit notre laboratoire, d'abord dans le cadre d'un étude princeps (Couvreur, Bruyninckx, \& Harmegnies, 1999), puis à la faveur d'une recherche exploratoire (Roland et al., 2016), et plus récemment dans le cadre d'une étude monographique approfondie (Roland, 2019) à investiguer le comportement, dans la parole de participants atteints de la MP, de sons caractérisés par la variation attendue de leur timbre au cours de leur production.

Au contraire de nombre d'autres langues, le français ne comporte cependant, dans son inventaire phonologique, aucune diphtongue. C'est donc ailleurs qu'il a fallu trouver des éléments dont la réalisation provoque des vocoïdes à timbre variable. Seule est utilisable ici la classe des "semi-consonnes » (parfois dénommées aussi " semi-voyelles »). Nous retiendrons ici les deux seuls phonèmes de ce type communs à tous les dialectes du français, à savoir $/ \mathrm{j} /$ et $/ \mathrm{w} /$. Leur intérêt pour notre questionnement provient du fait que, pour la réalisation de ces phonèmes, il y a lieu de produire un son vocoïde dont, transitoirement, le timbre approche (d'où la qualification d' " approximant » en littérature anglo-saxonne) soit celui de [u] (cas de /w/) soit celui de [i] (cas de /j/); la transition avec l'environnement immédiat doit s'effectuer sans solution de continuité depuis le timbre du segment précédent jusqu'à celui du segment suivant, en faisant continument évoluer (« glisser » - «to glide» en anglais, ce qui les fait souvent qualifier de " glides » dans la littérature phonétique ${ }^{i}$ ) le timbre tout au long de la production. Notre recherche investigue donc l'effet de la MP sur la capacité du locuteur à gérer la production de sons dont la variabilité est la spécificité et, dès lors, dont le contrôle des mouvements articulatoire est continuellement requis durant la production.

\section{Maladie de Parkinson}

Décrite pour la première fois en 1817 par le médecin anglais James Parkinson, la MP initialement dénommée shaking palsy - (Parkinson, 1817) est une maladie idiopathique chronique caractérisée par une dégénérescence d'un noyau de la substance noire qui agit sur les noyaux du striatum de la base du cerveau, le noyau caudé et le putamen. La MP est la deuxième maladie neurodégénérative la plus répandue après la maladie d'Alzheimer (Lee \& Gilbert, 2016; Mills-Joseph et al., 2018; Reich \& Savitt, 2019). Même si l'avancée en âge n'est pas la cause première de la maladie et que celle-ci débute moins fréquemment avant 50 ans (Korell \& Tanner, 2013), l'avancée en âge en reste un des principaux facteurs de risque (Darweesh et al., 2016). La cause du déclenchement de la maladie reste encore inconnue à ce jour, la majorité des cas étant sporadiques et multifactoriels (Ozsancak \& Auzou, 2005). De nombreuses recherches tentent toutefois d'identifier les origines de la maladie et les facteurs de prédiction de celle-ci. Plusieurs hypothèses sont ainsi évoquées, 
les plus connues étant les causes génétiques et environnementales (Fujioka \& Wszolek, 2012; Kalia \& Lang, 2015). A l'heure actuelle, aucune de ces causes ne semble pouvoir à elle seule expliquer totalement l'apparition de la maladie (Darweesh et al. 2016), celle-ci restant le plus fréquemment idiopathique (MPI). Le diagnostic clinique repose ainsi sur la présence de caractéristiques motrices de la maladie ainsi que sur l'absence de signes d'exclusion. Pour s'assister dans son diagnostic clinique, le neurologue dispose de plusieurs techniques et/ou outils et peut s'appuyer sur les critères établis par la UK Parkinson's Disease Society Brain Bank (UKPDSBB). Même s'ils ne sont pas systématiques voire ne font pas toujours consensus, des examens d'imagerie (principalement IRM et scintigraphie cérébrale au Dat Scan) peuvent être également pratiqués afin de confirmer un diagnostic de la MP, ceux-ci permettant d'observer les lésions du tronc cérébral et d'étudier la réduction des terminaisons nerveuses des neurones dopaminergiques afin d'exclure ou d'étayer des diagnostics différentiels (Kalia \& Lang, 2015).

Même s'ils ont souvent été considérés comme des troubles secondaires, voire d'apparition tardive, les troubles de la parole font partie de la symptomatologie de la MP. Ainsi, il apparait que 70 à $90 \%$ des personnes atteintes de la MP présentent ou développeront des troubles de la parole au cours de leur vie (par exemple: Hartelius \& Svensson, 1994; Pawlukowska, Szylińska, Kotlęga, Rotter, \& Nowacki, 2018). La dysarthrie est le terme générique utilisé pour désigner cet ensemble de troubles de la parole liés à des perturbations des commandes neuromusculaires des organes mis en jeu dans la production de la parole dont l'origine est une lésion du système nerveux central ou périphérique. Il s'agit donc d'un trouble de la parole d'origine neurologique qui se manifeste par une déficience motrice (Darley et al., 1969). Dès les travaux de Darley et collaborateurs, un effort a été réalisé afin de ne pas dissocier les troubles supralaryngés des troubles laryngés, faisant coexister dans la définition de la dysarthrie des troubles articulatoires, respiratoires, phonatoires, de résonance ainsi que des troubles prosodiques (Pinto, Ghio, Teston \& Viallet, 2010). De fait, la complexité des troubles dysarthriques réside dans leur potentialité à affecter toutes les composantes de la production de la parole (Kent, Weismer, Kent, Vorperian, \& Duffy, 1999).

Les dysarthries sont caractéristiques de certains symptômes associés aux grandes familles d'affections neurologiques ayant une influence plus ou moins grande sur la production de la parole. Dès 1969, Darley et al. tentent, à travers leur proposition de classification étiologique des dysarthries d'origine neurologique, de fournir une description précise de la dysarthrie présente dans la MP. Analysant la production de la parole dans la MP en y incluant l'articulation, la phonation et la résonance ainsi que la prosodie, ils identifient l'atteinte principale d'un cluster, celui de la prosodie, caractérisée par une insuffisance prosodique due à la réduction des mouvements articulatoires. D'autres altérations apparaissent, telles que des élocutions brèves, un débit de parole où la variabilité semble incontrôlée et une imprécision articulatoire, marquée principalement lors de la production de consonnes. Ces altérations résultent des mouvements répétitifs rapides mais de faibles amplitudes, caractéristiques de la MP. Des silences inappropriés ou une voix soufflée et rauque viennent compléter le tableau dysarthrique qui, en raison de la réduction de l'amplitude du mouvement dans la MP, est dit hypokinétique (Darley et al., 1969).

Dès la fin des années 70, Logemann et ses collaborateurs soulignaient la précision des symptômes décrits par Darley et al. (1969) mais regrettaient le fait que la description de ceux-ci sous-tend une uniformité des symptômes inhérents à la dysarthrie hypokinétique (Logemann, Fischer, Boshes \& Blonsky, 1978). Depuis, il est reconnu que la dysarthrie est un déficit encore plus complexe que ce qui avait été évoqué par Darley et ses collaborateurs, impliquant l'association de paramètres tels que l'imprécision des voyelles par exemple (Sapir, 2014). Il est également primordial de garder à l'esprit que la dysarthrie est un signe faisant partie d'un tableau clinique plus large relevant d'une symptomatologie globale découlant d'une pathologie dite du mouvement (Pinto et al., 2010). 


\section{Conséquences acoustiques de l'atteinte de la parole dans la dysarthrie hypokinétique}

Deux grandes catégories de sons de parole font l'objet d'évaluations acoustiques de l'articulation, les voyelles et les consonnes. Certes, il est communément accepté que les troubles dysarthriques vont entrainer une imprécision marquée des consonnes chez les locuteurs atteints de la MP (Darley, Aronson, \& Brown, 1969; Logemann \& Fisher, 1981). Même si elle a été évoquée plus tardivement, la production des voyelles tend également à être altérée (Mollaei, Shiller, Baum, \& Gracco, 2016; Skodda, Visser, \& Schlegel, 2011). Les voyelles sont principalement formées par les mouvements de plusieurs organes (langue, lèvre, mâchoire), ces mouvements modifiant les cavités de résonance (cavités buccale, nasale, pharyngale) et amplifiant dès lors certaines bandes de fréquence du spectre de la parole (Skodda, Visser \& Schlegel, 2011). Leur étude est ainsi réalisée depuis longtemps dans l'examen de la production de parole, les voyelles se distinguant les unes des autres au travers de patterns de résonance, appelés formants, dépendant de la configuration du conduit vocal lors de la production de sons de parole. Ces formants correspondent à des zones fréquentielles renforcées dans le signal sonore, chaque formant (premier formant $=$ $\mathrm{F} 1$, deuxième formant $=\mathrm{F} 2, \ldots$, nième formant $=\mathrm{Fn}$ ) correspondant à la nième fréquence de résonance amplifiée par la position des articulateurs. L'intérêt des formants est double en ce sens qu'ils permettent de distinguer les voyelles d'un point de vue acoustique et qu'ils renseignent sur la configuration des articulateurs. De fait, il est admis que la valeur du premier formant $(\mathrm{F} 1)$ renseigne sur le degré d'aperture tandis que la valeur du deuxième formant (F2) transmet quant à elle des informations sur le lieu d'articulation. La valeur du troisième formant (F3) peut également être employée pour obtenir des informations sur l'arrondissement des lèvres. Qui plus est, le passage d'un phonème à un autre entraine une modification des zones fréquentielles, nommée transition formantique (Ghio, 2007). Ainsi, étant donné qu'à la suite des modifications de la configuration du tractus vocal, les valeurs formantiques ne sont pas constantes d'un phonème à un autre, ces transitions formantiques représentent le passage de la cible articulatoire d'un premier son vers le suivant. La représentation sonagraphique peut être reportée, à partir des valeurs de F1 et de F2, dans un plan à deux dimensions représentant l'espace acoustique. L'étude des fréquences formantiques (exprimées en $\mathrm{Hz}$ ) fait ainsi partie des mesures acoustiques les plus fréquentes dans la MP, comme auprès de nombreuses autres populations de locuteurs, celleci permettant d'inférer des interprétations articulatoires à partir de données acoustiques (Kent \& Vorperian, 2018).

Les altérations liées à ces productions sont liées à une réduction de la force et de l'amplitude du mouvement articulatoire (Ackermann \& Ziegler, 1991; Duffy, 2012). Néanmoins, peu de travaux se sont concentrés sur la dynamique intra-segmentale du timbre imputable au contrôle des résonateurs (Goberman, Coelho, \& Robb, 2002) alors que son étude est tout à fait adaptée aux troubles associés à la MP. Moins encore se sont intéressés à la production de phonèmes requérant la réalisation de mouvements articulatoires intrasegmentaux très rapides et étroitement contrôlés.

\section{Evaluation des troubles dysarthriques dans la MP}

Longtemps, la dysarthrie hypokinétique a été évaluée perceptuellement, sur base de l'expertise du clinicien. Pour ce faire, il peut se baser sur l'échelle d'évaluation UPDRS qui permet de quantifier la progression de la maladie, et plus particulièrement sur l'exploitation de l'item « speech » de cette échelle décrivant la dysarthrie en cinq stades de sévérité croissante : 0 (parole normale), 1 (légère baisse de l'intonation et du volume), 2 (parole monotone, nettement perturbée mais compréhensible), 3 (perturbation marquée de la parole, difficilement compréhensible), 4 (parole inintelligible) (Pinto et al., 2010). 
A l'heure actuelle, les informations disponibles quant à la dysarthrie hypokinétique ainsi qu'à l'ensemble de la symptomatologie entourant la production de parole est basée tant sur des données perceptuelles, acoustiques, (neuro)physiologiques que de neuroimagerie. Le plus fréquemment, la dysarthrie est soit évaluée en milieu hospitalier à partir de méthodes cliniques, soit au moyen d'analyses acoustiques et expérimentales. Les choix posés en matière d'évaluation dépendent des questions que se posent le clinicien et/ou le chercheur. L'évaluation perceptuelle est appréciée en évaluation clinique en ce sens qu'elle permet un coût réduit d'un point de vue instrumental. Toutefois, ces techniques peuvent se heurter aux limites en matière de perception humaine, d'où l'importance de développer, depuis plusieurs décennies, des moyens instrumentaux d'évaluation de la parole, pouvant dès lors compléter et objectiver les évaluations perceptuelles (Kent et al., 1999).

\section{Méthodologie}

\subsection{Problématique de la recherche}

Comme nous l'avons évoqué dès l'introduction, notre recherche est partie d'un questionnement, celui du potentiel informatif du signal de parole produit par des locuteurs s'exprimant dans des situations de handicap comportant une dimension pathologique. Face à la diversité de troubles, nous avons fait choix d'axer nos recherches sur la MP. En effet, la MP est une maladie neurodégénérative présentant une large symptomatologie, au sein de laquelle se situent les troubles dysarthriques. Cette dysarthrie, dite hypokinétique dans la MP, a longtemps été considérée comme d'apparition tardive, en particulier en ce qui concerne ses répercussions sur l'intelligibilité de la parole, et plus largement sur la communication. Toutefois, cette considération d'une apparition tardive n'a pas toujours fait consensus et est, aujourd'hui, en partie réfutée. De fait, les réflexions menées sur l'évolution de la maladie (Braak et al., 2003) suggèrent que les troubles de la parole pourraient faire partie des premières manifestations de la MP (Sapir, 2014).

Nous avons décidé de nous centrer plus particulièrement sur les atteintes de la dysarthrie hypokinétique au niveau supraglottique, avec un intérêt particulier pour le contrôle des gestes articulatoires supralaryngés. De fait, la parole est un phénomène fluide, nécessitant des changements articulatoires rapides. La dynamique de la parole pose de grands défis du point de vue analytique, ce qui explique pourquoi les mesures réalisées dans une majorité d'études portent sur des portions déterminées de manière stricte, censées représenter les cibles visées et/ou des portions de signal stables (Kent \& Vorperian, 2018).

A partir d'une approche acoustique, notre objectif est d'observer la dynamique du mouvement et la production de voyelles stables ainsi que les altérations qui apparaissent dans la MP avec, comme hypothèse générale, que les personnes atteintes de la MP présentent des changements dans leur dynamique de mouvement articulatoire par rapport à des personnes sans pathologie ou maladie pouvant affecter la production de parole. Un de nos objectifs est également de mettre au jour des mesures acoustiques sensibles aux altérations de parole présentes précocement dans la MP. En effet, puisque la dysarthrie peut apparaitre de manière précoce dans la maladie, il nous semble opportun de tenter d'identifier des éléments permettant son identification, dans des perspectives de prise en charge voire de diagnostic précoce de la maladie. Dans le cas des analyses acoustiques, l'objectif est de quantifier les différences de parole entre des personnes atteintes de la MP et des locuteurs sains afin de comprendre l'impact du dysfonctionnement neurologique sur la production de la parole (Pinto, Chan, Guimaraes, Rothe-Neves \& Sadat, 2017), avec pour avantage une mesure non-invasive et peu coûteuse de nombreux paramètres de la production de la parole, permettant une inférence sur la fonction articulatoire (Dromey, Jang, \& Hollis, 2013).

Afin d'approcher l'étude de la dynamique articulatoire, nous allons nous centrer sur les glides étant donné qu'il s'agit de phonèmes qui, sur le plan phonétique, peuvent être 
considérés comme des vocoïdes dynamiques. Ce choix est donc effectué en raison de leur nature même puisque ce sont, en langue française, les rares phonèmes à nécessiter des mouvements continus des articulateurs supralaryngés (Couvreur et al., 1999). Qui plus est, les analyses sonagraphiques réalisées à partir de productions de logatomes de structure voyelle-glide-voyelle mettent en évidence la présence d'une structure formantique caractéristique. Plus précisément, c'est la potentielle informativité des glides qui nous intéresse, en partant d'une réflexion à caractère hypothétique : puisque les glides se caractérisent par une évolution continuelle de leur timbre durant leur production et puisque cette variation acoustique continue est le résultat direct de la variabilité de la géométrie des cavités résonantielles supraglottiques, l'analyse de la dynamique acoustique doit très directement renseigner sur les mouvements des articulateurs. De fait, comme le souligne Ghio (2007), la forme des transitions formantiques est un indicateur pertinent des configurations articulatoires.

Afin d'étudier cette dynamique articulatoire, plusieurs chercheurs se sont intéressés, entre autres, au calcul de pentes de F2. Les segments de parole les plus fréquemment étudiés incluent des diphtongues et des productions de syllabes consonne-voyelle (Lansford \& Liss, 2014; Martel-Sauvageau \& Tjaden, 2017). La production de diphtongues dans une perspective d'étude de la dynamique articulatoire est très pertinente en ce sens qu'il s'agit d'un phénomène dont le timbre varie en cours d'émission, consécutivement à la variation dans la position des articulateurs. Toutefois, ce type de son n'existe pas en langue française. Dès lors, nous avons choisi d'étudier par l'intermédiaire des glides le contrôle moteur chez les locuteurs atteints de la MP.

Peu d'études se sont intéressées à la production des glides. Logemann et Fisher (1981) suggèrent que ceux-ci sont relativement bien préservés dans la MP, contrairement à la production des consonnes qui est altérée. Dans leurs résultats, Gabbert-Downs, Garst, Dewey et Katz (2007) mettent quant à eux en évidence que, bien qu'ils maintiennent une variation proche de la norme dans la durée des syllabes, les personnes atteintes de la MP de leur étude $(n=9)$ n'arrivent pas à maintenir une synchronisation proportionnelle lors de la transition formantique du glide vers la voyelle. Selon ces auteurs, il existe plusieurs pistes explicatives plausibles pour expliquer cette difficulté lors de la transition formantique, dont notamment la distinction en ce qui concerne les lieux d'articulation du glide et de la voyelle considérée, ce qui pourrait expliquer les difficultés de contrôle du mouvement articulatoire lors de la production de ces séquences. Peu d'études sont néanmoins réalisées chez les personnes atteintes de la MP et encore moins le sont en langue française, ce qui motive dès lors notre intérêt pour ce type d'analyse. De fait, à notre connaissance, seule l'étude menée par Martel-Sauvageau et Tjaden (2017) a porté sur l'étude des glides chez des locuteurs atteints de la MP, en contexte francophone (québécois).

\subsection{Participants}

La participation de 98 locuteurs dont le français est la langue maternelle, répartis en deux groupes, a été sollicitée. Le premier groupe se compose de 63 participants (36 hommes et 27 femmes) atteints de la MP (gr. MP), âgés entre 38 et 85 ans au moment du recueil de données $(\mathrm{m}=70$ ans $)$. Le diagnostic de la maladie a été posé au minimum un an ant le recrutement jusqu'à une durée de maladie de 25 ans, la durée moyenne de la MP étant de 7 ans. Les participants présentent un éventail complet de l'ensemble des stades de la maladie tel que proposé par Hoehn et Yahr (1967) avec un effectif majoritairement présents des stades 1.5 à 4 de la maladie $(\mathrm{N}=59$ sur 63 participants). Suite à l'évaluation à partir de l'item parole de l'échelle d'évaluation UPDRS, vingt de nos participants ont été identifiés comme ne présentent pas de troubles dysarthriques. Le second groupe se compose quant à lui de 35 participants, 19 hommes et 16 femmes, âgés entre 41 et 84 ans ( $m=66$ ans), ne présentant pas de pathologies ni d'antécédents médicaux pouvant altérer la production de la parole (gr. témoin). 


\subsection{Tâches}

Après avoir été informés de l'étude en cours et avoir signé un formulaire de consentement éclairé stipulant l'enregistrement des productions orales, les participants ont été amenés à réaliser différentes tâches de production de parole dont :

- la production isolée des phonèmes / $\mathrm{a}, \mathrm{i}, \mathrm{u} /$ ( 5 répétitions par phonèmes) ;

- la production de logatomes de structure $\mathrm{V}_{\mathrm{a}, \mathrm{i}, \mathrm{u}} \mathrm{Gl}_{\mathrm{j}, \mathrm{w}} \mathrm{V}_{\mathrm{a}, \mathrm{i}, \mathrm{u}}$ (5 répétitions par logatome).

Dans cette étude, nous avons proposé à chaque participant de produire de manière isolée et brève les phonèmes $/ \mathrm{a}, \mathrm{i}, \mathrm{u} / \mathrm{afin}$ d'étudier les phonèmes inclus dans les logatomes par rapport aux monophtongues correspondantes, produits dans une situation similaire en matière de durée.

Afin de proposer un ensemble exhaustif de combinaisons V1GlV2, nous avons associé chacun des phonèmes /a, i, u/, en position initiale (V1) et finale (V2) aux glides /j, w/, seule exception faite de l'association des phonèmes correspondants à la cible du glide ([i] pour $/ \mathrm{j} /$ et $[\mathrm{u}]$ pour $/ \mathrm{w} /$ ). Nous avons obtenu une liste de huit logatomes (tableau 1), quatre logatomes incluant le glide $/ \mathrm{j} /$ et quatre logatomes incluant le glide $/ \mathrm{w} /$.

Tableau 1. Liste des logatomes de structure V1GlV2 à l'étude.

\begin{tabular}{|l|l|l|l|}
\hline$V 1$ & /a/ & /i/ & $/ \mathrm{u} /$ \\
\hline$/ \mathrm{a} /$ & \begin{tabular}{llll|} 
/aja/ \\
/awa/
\end{tabular} & /awi/ & /aju/ \\
\hline$/ \mathrm{i} /$ & /iwa/ & /iwi/ & \\
\hline$/ \mathrm{u} / /$ & $/$ uja/ & & $/$ uju/ \\
\hline
\end{tabular}

Ces tâches de production nous permettent de tester l'aptitude au contrôle des mouvements fins des articulateurs supralaryngés oraux et de comparer les données des personnes du groupe MP à celles de personnes du groupe témoin. Chaque participant (gr. MP et gr. témoin) a ainsi réalisé quinze productions de voyelles en isolation et quarante productions de logatomes de structure V1GlV2, ce qui nous permet, au total, l'étude de 5390 productions de parole statique et dynamique. Ces productions de parole ont toutes été enregistrées en une seule passation par sujet, à l'aide d'un enregistreur portatif ZOOM H5. Tous les participants atteints de la MP ont été enregistrés dans une pièce calme au sein de l'hôpital où ils étaient suivis, à l'occasion de visites de contrôle. Les sons de parole recueillis sont le résultat d'injonctions de production à partir de versions écrites des productions à réaliser, présentées sur un écran d'ordinateur portable placé face aux participants, l'utilisation d'un support informatique nous permettant d'ajuster la taille de l'écriture aux besoins des participants. Tous les participants ayant été enregistrés dans la même pièce, la même disposition du matériel de présentation et d'enregistrement a été instaurée.

\subsection{Hypothèses de recherche}

Dans cette étude, notre objectif est d'explorer la dynamique des articulateurs supralaryngés, à travers l'étude acoustique de la production de sons de parole dans un contexte dynamique incluant des glides. Notre hypothèse porte sur le fait que les personnes atteintes de la MP présentent une dynamique acoustique différente de celle de personnes sans pathologies pouvant affecter la production de la parole. Disposant d'une riche quantité d'informations sur nos locuteurs, nous posons également comme hypothèse que certains indicateurs de la MP - en particulier le degré de sévérité de la dysarthrie - peuvent permettre de dégager des pistes explicatives des altérations apparaissant lors de la production de parole. A partir de nos résultats, nous tenterons de vérifier les hypothèses suivantes :

- la production de logatomes de structure V1GIV2 se réalise en préservant un timbre pour le centre du glide proche de celui de la cible acoustique, au détriment du timbre des 
phonèmes aux extrémités du logatome (V1-V2). La précision articulatoire se réaliserait dès lors de manière sélective, certaines cibles étant préservées au détriment des autres ;

- l'ampleur des transitions formantiques est réduite chez les locuteurs atteints de la MP tant lors de la transition de la V1 au glide que lors de la transition du glide à la V2 afin de préserver le tempo de parole, suggérant un phénomène d'undershoot ;

- des altérations dans la production des logatomes apparaissent chez des locuteurs ne présentant pas ou peu de troubles dysarthriques, permettant l'identification de phénomènes de nature infraclinique.

\subsection{Analyses acoustiques}

A partir des données et des échantillons de parole recueillis, nous effectuerons des analyses acoustiques au moyen du logiciel PRAAT ainsi que des analyses statistiques à partir du logiciel statistique SPSS (IBM SPSS Statistics 25). Les analyses acoustiques ont consisté en l'extraction des valeurs de $1^{\mathrm{er}}$ et de $2^{\text {ème }}$ formants ainsi que des mesures de durée pour toutes les productions recueillies dans chacun des deux groupes. Nous avons extrait manuellement ces mesures au moyen de l'algorithme de suivi formantique ainsi qu'à partir de l'examen spectrographique fournis par le logiciel PRAAT. Nous avons ensuite effectué des vérifications et si nécessaire, des corrections.

Les logatomes produits par chaque participant ont permis le recueil d'informations variées en vue de caractériser la dynamique acoustique de production de la parole. Au vu de la structure des logatomes, les valeurs formantiques ont été extraites à trois endroits de la production : à l'entame du logatome (F1 et F2 de la voyelle 1 - départ), au centre du glide (F1 et F2 du glide - extremum ${ }^{\mathrm{ii}}$ ) et au terme de la production (F1 et F2 de la voyelle 2 arrivée). Le centre du glide a été déterminé comme étant le point d'extremum des traces formantiques apparaissant sur le tracé spectrographique (Fig. 1)

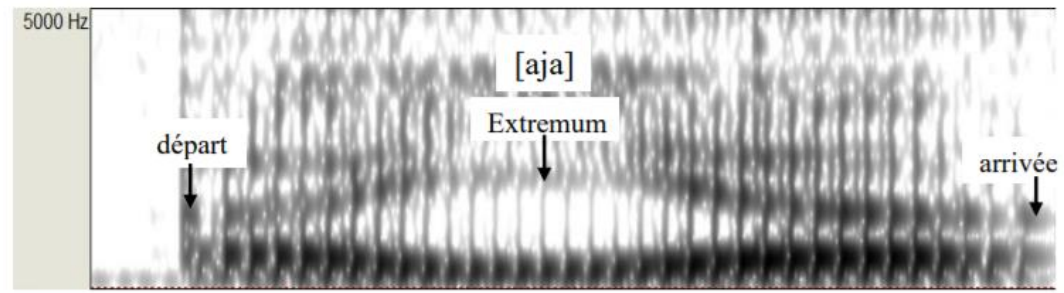

Fig. 1. Représentation spectrographique de la réalisation d'un logatome de type /aja/.

Ces mesures ont ensuite été considérées comme objet du plan F1/F2 (Fig. 2). Les tracés curvilinéaires apparaissant sur la figure représentent l'illustration de la variation constante du timbre en cours de production, déterminée par ce que nous nommerons la distance " aller » - de la V1 du logatome au centre du glide - et la distance « retour » - du centre du glide à la V2 du logatome. 


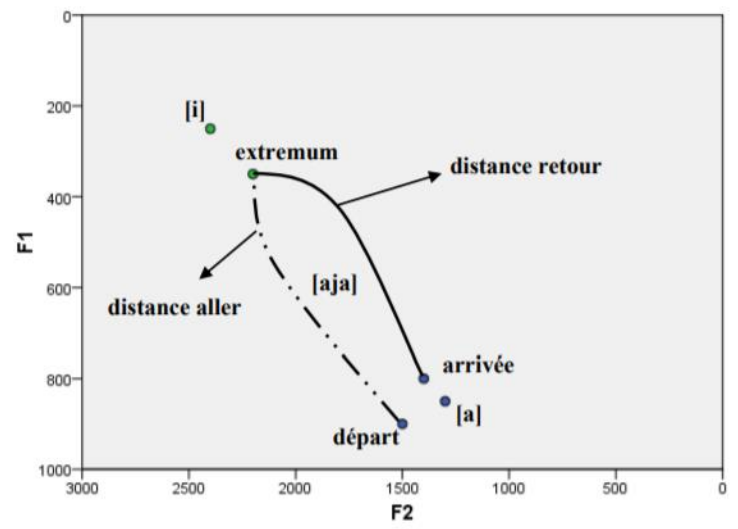

Fig. 2. Représentation d'un logatome de type /aja/ considéré dans un plan F1/F2.

L'extraction de ces valeurs nous a permis le calcul de diverses distances euclidiennes entre deux points $\mathrm{a}$ et $\mathrm{b}$ dans le plan $\mathrm{F} 1 / \mathrm{F} 2$ à partir de la formule suivante :

$$
\text { Distance }(a, b)=\sqrt{ }\left[(F 2 a-F 2 b)^{2}+(F 1 a-F 1 b)^{2}\right]
$$

Celles que nous présenterons ici sont de deux ordres :

- des distances euclidiennes nous informant sur l'ampleur des transitions formantiques au sein du logatome, à partir de distances dites « aller » et « retour »;

- des distances euclidiennes nous informant sur la localisation des phonèmes inclus dans un logatome de structure V1GlV2 dans le plan F1/F2 par rapport à ceux produits isolément lors de la production des voyelles isolées - dénommées par la suite distances à la voyelle de référence (à titre illustratif, distance euclidienne entre le /a/ lors de la production du logatome /aja/ et le /a/ produit de manière isolée.

L'extraction et le calcul de distances euclidiennes nous permettent ainsi d'obtenir des informations quant à l'ampleur des transitions formantiques au sein du logatome ainsi que des informations concernant la distance séparant les phonèmes produits inclus dans un logatome de structure V1GlV2 et les monophtongues correspondantes.

\section{Résultats}

\subsection{Amplitude des transitions formantiques dans le plan F1/F2}

\subsubsection{Distances euclidiennes}

Nos analyses statistiques (U de Mann-Whitney) mettent en évidence des différences significatives entre nos deux groupes de participants lors du passage de la première voyelle du logatome à la cible du glide $(\mathrm{U}=86.288, \mathrm{p}<0.001)$ ainsi que lors de la transition de la cible du glide à la cible de la seconde voyelle $(\mathrm{U}=86.214, \mathrm{p}<0.001)$, suggérant de mouvements articulatoires de plus faible amplitude chez les locuteurs du groupe MP. Au vu de ces différences, l'examen logatome par logatome des distances parcourues, entre le point de départ de la production et le point d'extremum d'une part (distance « aller ») (Fig. 3), puis, d'autre part, entre ce même point d'extremum et le point d'arrivée de la production (distance « retour ») (Fig. 4) nous apparait comme pertinent afin d'observer les possibles différences en fonction des cibles acoustiques à atteindre. Afin de faciliter la lecture des graphiques, nous présentons d'abord les logatomes se composant des mêmes voyelles initiale et finale, puis ceux où V1 et V2 diffèrent. 

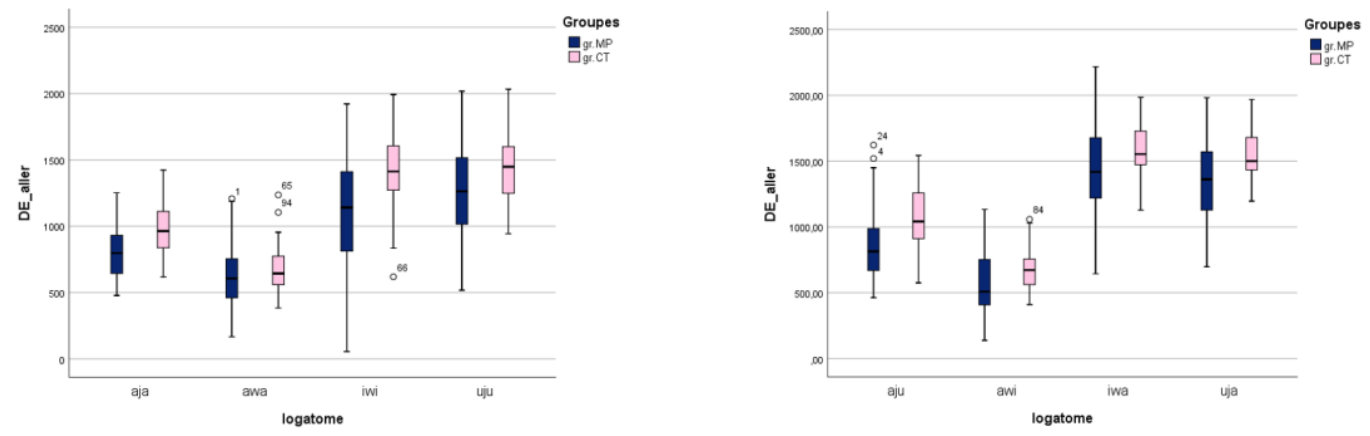

Fig. 3. Distance «aller» au sein des deux groupes selon les logatomes produits, comportant les mêmes voyelles initiales et finales (gauche) et des voyelles initiales et finales différentes (droite).
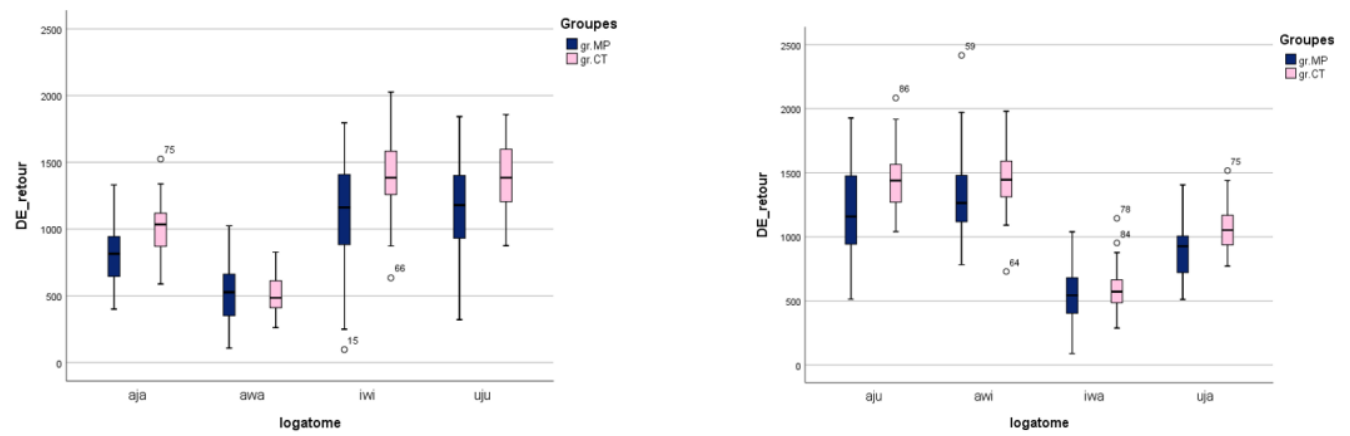

Fig. 4. Distance « retour» au sein des deux groupes selon les logatomes produits, comportant des voyelles initiale et finale identiques (gauche) ou différentes (droite).

Dans les deux situations, une moindre ampleur des transitions formantiques peut être remarquée chez les participants du groupe MP, tant pour les logatomes composés de voyelles identiques en début et fin de logatomes que de voyelles différentes. Ces observations nous permettent de constater qu'un grand nombre de différences dans l'amplitude du mouvement semble concerner le passage des phonèmes /i/ ou /u/ vers les glides $/ \mathrm{w} /$ ou $/ \mathrm{j} /$ et inversement, soutenant de ce fait notre hypothèse d'une difficulté de modification rapide du lieu d'articulation chez les locuteurs atteints de la MP.

\subsubsection{Pentes formantiques}

Le calcul des pentes de F2 a été réalisé afin de raffiner nos observations concernant la dynamique des gestes articulatoires à travers l'étude des transitions formantiques. Nous les avons calculées à partir des trois endroits où ont été extraites les valeurs formantiques : à l'entame de la production (V1), au centre du glide et en fin de production (V2). Cela nous a permis d'obtenir une première pente formantique concernant le passage de V1 au glide ainsi qu'une seconde permettant le passage du glide à V2.

Afin de procéder à des comparaisons entre nos deux groupes de locuteurs, nous avons tout d'abord sélectionné les logatomes incluant le glide /w/. Nous constatons des différences significatives entre nos deux groupes lors du calcul de la pente «aller » $(\mathrm{U}=$ 3058, p < 0.001), la pente de F2 étant moindre chez les locuteurs du groupe MP (Fig. 5). 


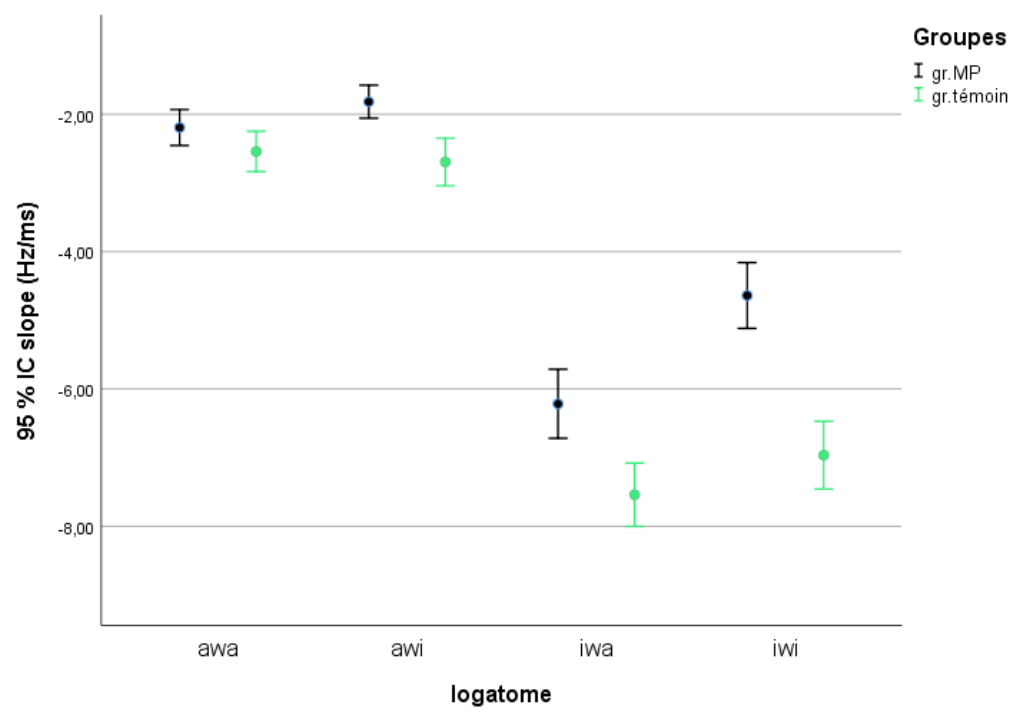

Fig. 5. Valeurs des pentes de F2 «aller» issues des logatomes incluant le glide /w/, en fonction des groupes de locuteurs.

Notons qu'ici, la pente est négative étant donné que nous avons pris en considération le glide /w/ qui entraine une transition formantique descendante, qu'il soit associé au phonème /a/ ou $/ \mathrm{i} /$.

Des constats similaires apparaissent concernant les pentes de F2 des logatomes incluant le glide $/ \mathrm{j} /(\mathrm{U}=6982, \mathrm{p}<0.001)$, la pente étant réduite chez les locuteurs atteints de la MP (Fig. 6). Notons qu'ici la pente est positive étant donné que nous sommes face à une transition formantique montante.

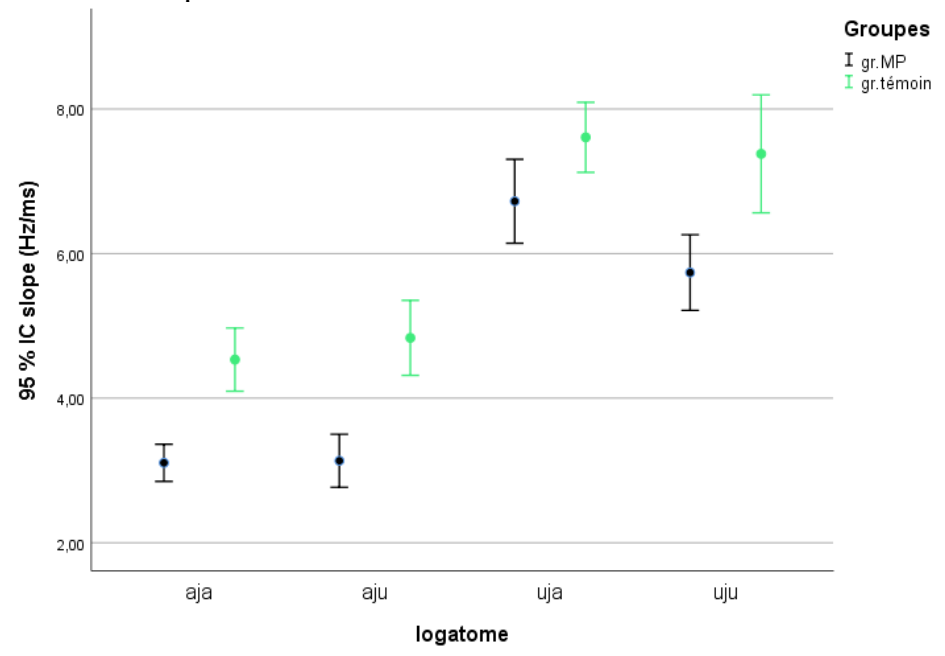

Fig. 6. Valeurs des pentes de $\mathrm{F} 2$ «aller» issues des logatomes incluant le glide /j/, en fonction des groupes de locuteurs.

Les mêmes constats apparaissent lors du calcul de la pente F2 concernant la deuxième partie du logatome (du glide à V2) et ce, tant pour le glide $/ \mathrm{w} /(\mathrm{U}=6117, \mathrm{p}<0.001)$ que le glide $/ \mathrm{j} /(\mathrm{U}=1241, \mathrm{p}<0.001)$.

\subsubsection{Phénomènes de nature infraclinique}

Suite à l'identification d'une diminution de l'amplitude des transitions formantiques, nous nous sommes interrogés sur le caractère potentiellement infraclinique de nos observations. 
Pour ce faire, nous avons comparé les productions des locuteurs atteints de la MP considérés comme non dysarthriques $(\mathrm{N}=20)$ à celles des locuteurs du groupe témoin.

En ce qui concerne l'amplitude des mouvements articulatoires, nos analyses nous permettent de constater que les distances « aller» (Fig. 7) et « retour» (Fig. 8) sont réduites chez les locuteurs atteints de la MP (respectivement, $U=25.932, p=0.006$ et $U=25.814$, $p$ $=0.008$ ).

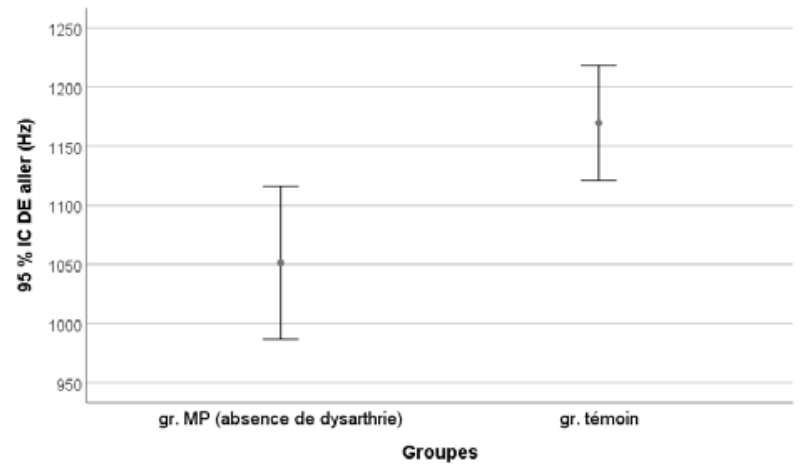

Fig. 7. Distance «aller» pour les locuteurs du groupe MP ne présentant pas de dysarthrie (gauche) et pour les locuteurs du groupe témoin (droite).

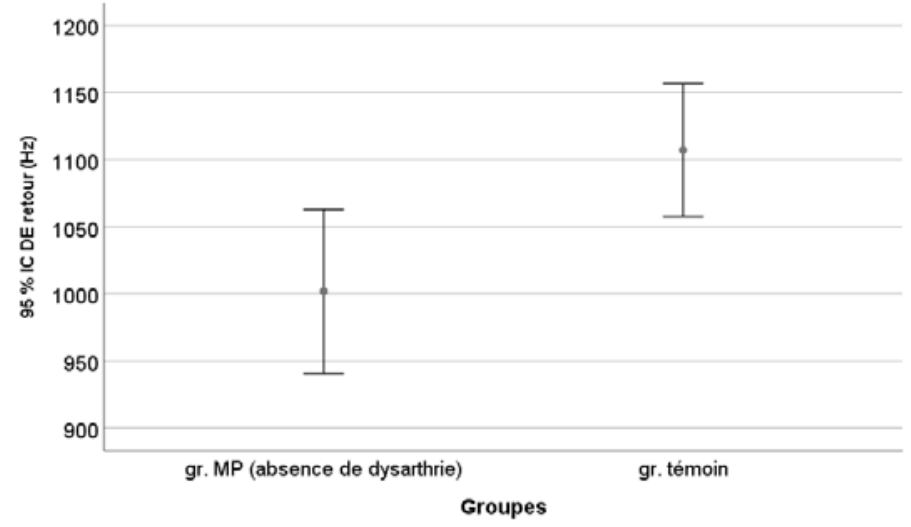

Fig. 8. Distance « retour » pour les locuteurs du groupe MP ne présentant pas de dysarthrie (gauche) et les locuteurs du groupe témoin (droite).

Nos analyses mettent également en évidence des différences significatives entre ces deux groupes (MP non dysarthriques et sujets témoins), tant pour les logatomes incluant le glide / $\mathrm{w} /$ que le glide $/ \mathrm{j}$ / et ce, tant pour les pentes formantiques marquant le passage de $\mathrm{V} 1$ au glide (respectivement, $\mathrm{U}=1297, \mathrm{p}=0.001$ et $\mathrm{U}=3037, \mathrm{p}<0.001$ ) que celles allant du glide à V2 (respectivement, $\mathrm{U}=2674, \mathrm{p}<0.001$ et $\mathrm{U}=3092, \mathrm{p}<0.001$ ).

Nous constatons dès lors que tant les distances euclidiennes «aller» et « retour » que les pentes de F2 sont toujours moindres chez les locuteurs atteints de la MP comparativement aux productions des locuteurs du groupe témoin, et ce même lorsque les participants du groupe MP ne présentent pas encore de troubles considérés comme dysarthriques.

\subsection{Dispersion des logatomes dans le plan F1/F2}

\subsubsection{Distances euclidiennes}

À la suite de ces observations concernant l'amplitude des transitions formantiques, nous nous sommes interrogés sur la proximité entre les monophtongues produites de manière stable et isolée et les phonèmes composant les logatomes en début (V1), milieu (glide) et fin de production (V2). En effet, la diminution de l'amplitude articulatoire des mouvements 
peut avoir plusieurs causes qu'il convient d'explorer. Pour ce faire, nous avons procédé en calculant les distances euclidiennes entre les phonèmes inclus dans les logatomes et les monophtongues correspondantes.

En premier lieu, nous avons considéré la distance séparant la V1 de chaque logatome produit et la voyelle de référence correspondante. La comparaison entre les productions des locuteurs du groupe MP et du groupe témoin ne permet pas de mettre en évidence des différences significatives entre les deux groupes (Fig. 64), ce qui suggère une préservation du timbre de la voyelle initiale. Le même constat apparait lorsque nous questionnons la distance entre la section finale des logatomes et les monophtongues correspondantes.

A contrario, notre analyse montre que l'éloignement du point correspondant à la monophtongue est significativement plus grand pour la cible du glide dans les logatomes produits par les participants atteints de la MP $(U=61.769, \mathrm{p}=0.004)$ (Fig. 9).

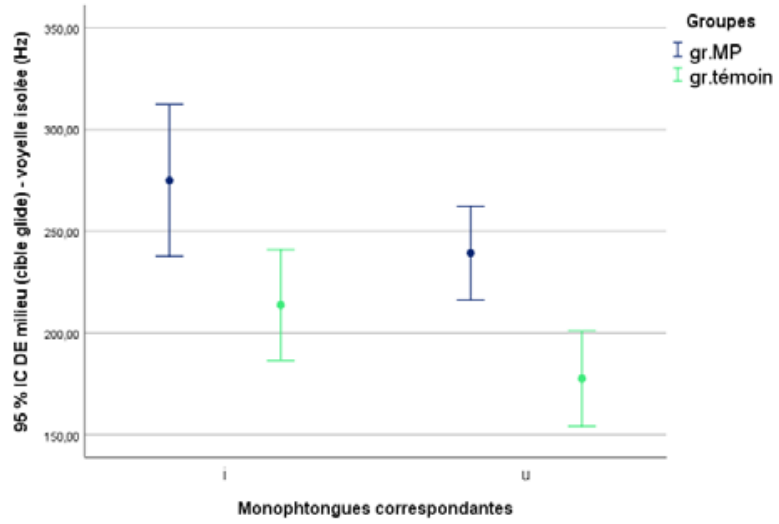

Fig. 9. Distance euclidienne à la référence - cible du glide pour chaque groupe de locuteurs selon la monophtongue correspondante.

Ce résultat suggère que les locuteurs atteints de la MP produisent des timbres plus influencés par la voyelle initiale ou finale du logatome que par la cible du glide.

\subsubsection{Phénomènes de nature infraclinique}

A nouveau, nous nous sommes interrogés sur les différences apparaissant entre les productions des locuteurs atteints de la MP considérés comme non dysarthriques et celles de notre groupe témoin. Les analyses menées révèlent des différences significatives uniquement pour l'éloignement du point correspondant à la monophtongue ciblée par le glide $(\mathrm{U}=23.533, \mathrm{p}=0.003)$ (Fig. 10), là où les timbres des voyelles initiales et finales restent préservés. Cela met en évidence que nos constats précédents sont déjà présents chez des locuteurs non atteints sur le plan arthrique, comparativement à des locuteurs témoins. 


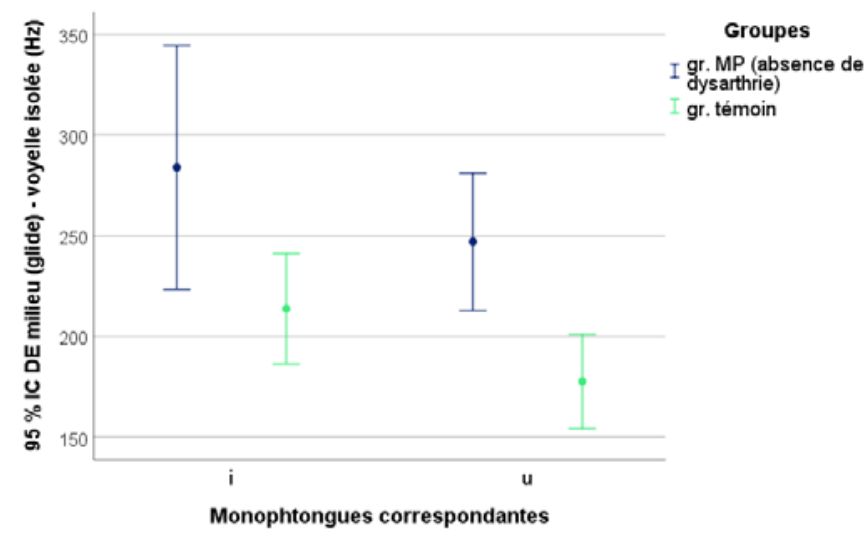

Fig. 10. Distance euclidienne à la référence - cible du glide pour chaque groupe de locuteurs (MP sans dysarthrie et témoins) selon la monophtongue correspondante.

\section{Discussion des résultats}

Nos analyses, sur base de la comparaison des variations de timbre, nous ont permis de constater que les mouvements réalisés lors de la production des logatomes sont de moins grande amplitude pour les participants du groupe MP comparativement aux productions du groupe témoin, et ce quel que soit le glide considéré ou la similitude/différence entre les voyelles initiales et finales composant le logatome. Nous y avons constaté une variabilité plus marquée chez les locuteurs atteints de la MP lors de la production de logatomes incluant le passage des voyelles $/ \mathrm{i}, \mathrm{u} /$ aux cibles des glides $/ \mathrm{w}, \mathrm{j} /$ ainsi que de ces cibles aux voyelles $/ \mathrm{i}, \mathrm{u} /$. Ceci nous amène à nous questionner sur la possible difficulté qu'induisent les transitions permettant le passage d'une cible à l'autre. En effet, il s'agit ici de phonèmes nécessitant des transitions rapides et importantes de la géométrie des cavités supraglottiques. Ces constats nous amènent à suggérer que la réduction de l'amplitude du mouvement peut résulter d'une difficulté à modifier rapidement la configuration des articulateurs supralaryngés. Cette inférence articulatoire à partir de données acoustiques nous apparait renforcée par le fait que ces difficultés s'intensifient avec la progression de l'atteinte dysarthrique, suggérant une aggravation de la réduction des gestes articulatoires.

En ce qui concerne la localisation dans le plan formantique F1/F2, il apparait que l'éloignement du point correspondant à la monophtongue est plus grand pour la cible du glide qu'il ne l'est pour les voyelles initiales et finales des logatomes. Plus qu'une stratégie hypoarticulatoire, nous nous questionnons ici sur la possible influence des constats réalisés en ce qui concerne l'amplitude du mouvement. En effet, nos analyses nous amenant à suggérer des difficultés concernant la modification du lieu d'articulation, notre hypothèse est que la cible des glides se distancie des voyelles correspondantes à la suite des difficultés rencontrées par les locuteurs atteints de la MP pour réaliser une modification rapide du positionnement des articulateurs.

Dans cette étude, nous avons également pris en considération le degré de sévérité de la dysarthrie, et plus particulièrement l'absence de troubles dysarthriques. Cet indicateur personnel nous a permis une meilleure compréhension de nos résultats. Ainsi, nous avons constaté une influence du degré de sévérité de la dysarthrie lors de la production des logatomes de structure V1GlV2 avec, notamment, une influence sur l'amplitude du mouvement articulatoire. Ces résultats suggèrent la présence d'une difficulté de modification rapide du lieu d'articulation, et dès lors possiblement des gestes articulatoires, chez les locuteurs atteints de la MP. Cela est notamment cohérent avec les résultats de Kim et al. (2009) qui ont identifié des pentes formantiques nettement inférieures chez des locuteurs atteints de la MP lors de la production de diphtongues nécessitant des modifications rapides et importantes de la géométrie du tractus vocal. Martel-Sauvageau et 
Tjaden (2017) évoquent également une sensibilité particulière à la dysarthrie des éléments phonétiques nécessitant des modifications relativement rapides et importantes du tractus vocal. Réaliser des gestes articulatoires précis est une des étapes essentielles à la production de la parole, cela rendant les productions émises intelligibles à un interlocuteur. Dès lors, nous pouvons nous questionner sur les répercussions des difficultés constatées sur l'intelligibilité de la parole de locuteurs atteints de la MP.

Pour donner suite à ces constations, nous nous sommes interrogés plus précisément sur la présence de phénomènes de nature infraclinique, les participants atteints de la MP présentant différents degrés de dysarthrie. Pour certains, une absence de dysarthrie était constatée $(\mathrm{N}=20)$. Nous nous sommes dès lors interrogés sur les potentielles différences entre les productions orales de ce groupe de locuteurs atteints de la MP et celles des locuteurs du groupe témoin. Nos résultats vont dans le sens de l'identification d'altérations précoces chez ces personnes atteintes de la MP. En effet, les analyses effectuées nous permettent d'identifier des différences déjà présentes entre nos deux groupes de locuteurs lors de la production de mouvements articulatoires dynamiques, ce qui nous suggère l'identification de phénomènes de nature infraclinique. Etant donné le nombre de personnes qui connaitront au cours de leur maladie des troubles dysarthriques, identifier précocement ce symptôme nous semble revêtir un caractère crucial en vue d'une prise en charge précoce.

\section{Conclusions}

Dans cette étude, les participants présentaient différents degrés de sévérité de dysarthrie (légère, modérée et sévère). Une partie de ce groupe était évalué comme exempt de dysarthrie hypokinétique. De ce fait, il nous a semblé opportun de comparer les résultats de ce dernier sous-groupe (participants atteints de la MP et non dysarthriques) avec ceux du groupe témoin. Comme vu précédemment, ces analyses ont révélé des différences significatives entre nos deux groupes de participants, tant sur des données statiques qui n'ont pu être abordées ici (exploitation de l'espace vocalique) que sur des données dynamiques (production de logatomes incluant des glides). Ces résultats viennent donc affirmer le caractère infraclinique des phénomènes mis au jour.

A notre connaissance, aucune étude n'avait été menée en ce sens auprès de participants atteints de la MP mais non atteints sur le plan arthrique. Outre l'apport de ces constats d'un point de vue de la recherche en phonétique, nous considérons ces données comme particulièrement pertinentes en perspective du développement d'outils de dépistage précoce de la dysarthrie, voire de techniques d'accompagnement de la rééducation logopédique. Dès lors, recueillir des données auprès de participants pour qui le diagnostic vient d'être posé (MP « natifs ») pourrait également nous fournir des informations très pertinentes. Ce type de recueil de données pourrait, entre autres, nous permettre d'observer des altérations très précocement dans la maladie, voire de développer de nouveaux indicateurs venant enrichir le diagnostic clinique de la maladie.

Une réflexion quant aux différentes tâches permettant le recueil des productions de parole est également utile. Dans notre étude, nous avons eu recours à des productions hors contexte communicationnel, le recours aux phrases porteuses ayant été écarté en vue d'éviter le biais d'hypoarticulation susceptible de survenir dans la MP lorsque le sujet est amené à réaliser des productions longues (Martel-Sauvageau et al., 2015). A l'avenir, il sera intéressant d'investiguer également ces productions. Confortés quant au potentiel informatif de l'étude des glides, il nous semble que leurs études dans d'autres situations de production auraient tout son sens (à titre illustratif, à partir d'une description d'image dans laquelle plusieurs éléments permettraient la production de mots incluant des glides). 


\section{Références bibliographiques}

Ackermann, H., \& Ziegler, W. (1991). Articulatory deficits in parkinsonian dysarthria: an acoustic analysis. Journal of Neurology, Neurosurgery \& Psychiatry, 54(12), 1093-1098. https://doi.org/10.1136/jnnp.54.12.1093

Braak, H., Tredici, K. D., Rüb, U., de Vos, R. A. I., Jansen Steur, E. N. H., \& Braak, E. (2003). Staging of brain pathology related to sporadic Parkinson's disease. Neurobiology of Aging, 24(2), 197-211. https://doi.org/10.1016/S0197-4580(02)00065-9

Couvreur, N., Bruyninckx, M., \& Harmegnies, B. (1999). Effects of parkinsonian symptoms on voiced palatals. Proceedings of 14th International Congress of Phonetic Sciences, 1, 831-834. San Francisco.

Darley, F. L., Aronson, A. E., \& Brown, J. R. (1969). Clusters of Deviant Speech Dimensions in the Dysarthrias. Journal of Speech and Hearing Research, 12(3), 462-496. https://doi.org/10.1044/jshr.1203.462

Darweesh, S. K. L., Verlinden, V. J. A., Adams, H. H. H., Uitterlinden, A. G., Hofman, A., Stricker, B. H., ... Ikram, M. A. (2016). Genetic risk of Parkinson's disease in the general population. Parkinsonism \& Related Disorders, 29, 54-59. https://doi.org/10.1016/j.parkreldis.2016.05.030

Dromey, C., Jang, G.-O., \& Hollis, K. (2013). Assessing correlations between lingual movements and formants. Speech Communication, 55(2), 315-328. https://doi.org/10.1016/j.specom.2012.09.001

Duffy, J. R. (2012). Motor speech disorders : substrates, differential diagnosis and management (3e éd.). St Louis: Mosby.

Fujioka, S., \& Wszolek, Z. K. (2012). Update on Genetics of Parkinsonism. Neurodegenerative Diseases, 10(1-4), 257-260. https://doi.org/10.1159/000334285

Ghio, A. (2007). L'évaluation acoustique. In Les dysarthries (P. Auzou, V. Rolland-Monnoury, S. Pinto, C. Özsancak, p. 236-247). Marseille: Solal.

Goberman, A., Coelho, C., \& Robb, M. (2002). Phonatory characteristics of Parkinsonian speech before and after morning medication: the ON and OFF states. Journal of Communication Disorders, 35(3), 217-239. https://doi.org/10.1016/S0021-9924(01)00072-7

Hartelius, L., \& Svensson, P. (1994). Speech and swallowing symptoms associated with Parkinson's Disease and Multiple Sclerosis: A survey. Folia Phoniatrica et Logopaedica, 46, 9-17.

Hoehn, M. M., \& Yahr, M. D. (1967). Parkinsonism: onset, progression, and mortality. Neurology, 17(5), 427-442. https://doi.org/10.1212/WNL.17.5.427

Kalia, L. V., \& Lang, A. E. (2015). Parkinson's disease. The Lancet, 386(9996), 896-912. https://doi.org/10.1016/S0140-6736(14)61393-3

Kent, R. D., \& Vorperian, H. K. (2018). Static measurements of vowel formant frequencies and bandwidths: A review. Journal of Communication Disorders, 74, 74-97. https://doi.org/10.1016/j.jcomdis.2018.05.004

Kent, R. D., Weismer, G., Kent, J. F., Vorperian, H. K., \& Duffy, J. R. (1999). Acoustic studies of dysarthric speech: methods, progress, and potential. Journal of Communicative Dirsorders, 32, 141-186.

Korell, M., \& Tanner, C. M. (2013). Epidemiology of Parkinson's Disease: An overview. In R. F. Pfeiffer, Z. K. Wszolek, \& M. Ebadi, Parkinson's Disease (2e éd.). Boca Raton: Taylor \& Francis Group.

Lansford, K. L., \& Liss, J. M. (2014). Vowel Acoustics in Dysarthria: Speech Disorder Diagnosis and Classification. Journal of Speech, Language, and Hearing Research, 57(1), 57-67. https://doi.org/10.1044/1092-4388(2013/12-0262)

Lee, A., \& Gilbert, R. M. (2016). Epidemiology of Parkinson Disease. Neurologic Clinics, 34(4), 955-965. https://doi.org/10.1016/j.ncl.2016.06.012 
Logemann, J. A., \& Fisher, H. B. (1981). Vocal Tract Control in Parkinson's Disease. Journal of Speech and Hearing Disorders, 46(4), 348-352. https://doi.org/10.1044/jshd.4604.348

Logemann, J. A., Fisher, H. B., Boshes, B., \& Blonsky, E. R. (1978). Frequency and Cooccurrence of Vocal Tract Dysfunctions in the Speech of a Large Sample of Parkinson Patients. Journal of Speech and Hearing Disorders, 43(1), 47-57. https://doi.org/10.1044/jshd.4301.47

Martel-Sauvageau, V., \& Tjaden, K. (2017). Vocalic transitions as markers of speech acoustic changes with STN-DBS in Parkinson's Disease. Journal of Communication Disorders, 70, 1-11. https://doi.org/10.1016/j.jcomdis.2017.10.001

Mills-Joseph, R., Krishna, V., Deogaonkar, M., \& Rezai, A. R. (2018). Deep Brain Stimulation in Parkinson's Disease. In Neuromodulation (p. 911-917). https://doi.org/10.1016/B978-0-12805353-9.00074-7

Mollaei, F., Shiller, D. M., Baum, S. R., \& Gracco, V. L. (2016). Sensorimotor control of vocal pitch and formant frequencies in Parkinson's disease. Brain Research, 1646, 269-277. https://doi.org/10.1016/j.brainres.2016.06.013

Ozsancak, C., \& Auzou, P. (2005). Les troubles de la parole et de la déglutition dans la maladie de Parkinson. Marseille: Solal.

Parkinson, J. (1817). An essay on the Shaking Palsy. Consulté à l'adresse https://archive.org/details/essayonshakingpa00parkuoft

Pawlukowska, W., Szylińska, A., Kotlęga, D., Rotter, I., \& Nowacki, P. (2018). Differences between Subjective and Objective Assessment of Speech Deficiency in Parkinson Disease. Journal of Voice, 32(6), 715-722. https://doi.org/10.1016/j.jvoice.2017.08.018

Pinto, S., Chan, A., Guimarães, I., Rothe-Neves, R., \& Sadat, J. (2017). A cross-linguistic perspective to the study of dysarthria in Parkinson's disease. Journal of Phonetics, 64, 156-167. https://doi.org/10.1016/j.wocn.2017.01.009

Pinto, S., Ghio, A., Teston, B., \& Viallet, F. (2010). La dysarthrie au cours de la maladie de Parkinson. Histoire naturelle de ses composantes: dysphonie, dysprosodie et dysarthrie. Revue Neurologique, 166(10), 800-810. https://doi.org/10.1016/j.neurol.2010.07.005

Reich, S. G., \& Savitt, J. M. (2019). Parkinson's Disease. Medical Clinics of North America, 103(2), 337-350. https://doi.org/10.1016/j.mcna.2018.10.014

Roland, V. (2019). Production de la parole dans la maladie de Parkinson. Aspects phonétiques, répercussions communicationnelles et perspectives de prise en charge (Thèse de doctorat). Université de Mons

Roland, V., Delvaux, V., Huet, K., Piccaluga, M., Haelewyck, M. C., \& Harmegnies, B. (2016). Dynamique phonétique et contrôle moteur dans la maladie de Parkinson : analyse du contrôle de la production des glides. Actes de la conférence conjointe JEP-TALN-RECITAL 2016, 1, 211219. Paris.

Sapir, S. (2014). Multiple Factors Are Involved in the Dysarthria Associated With Parkinson's Disease: A Review With Implications for Clinical Practice and Research. Journal of Speech, Language, and Hearing Research, 57(4), 1330-1343. https://doi.org/10.1044/2014_JSLHR-S-130039

Skodda, S., Visser, W., \& Schlegel, U. (2011). Vowel Articulation in Parkinson's Disease. Journal of Voice, 25(4), 467-472. https://doi.org/10.1016/j.jvoice.2010.01.009

\footnotetext{
${ }^{\mathrm{i}}$ C'est la terminologie que nous utiliserons dans notre contribution pour évoquer les « vocoïdes à timbre dynamique $»$.

ii Le terme extremum est ici utilisé pour faire référence au moment d'inversion de la pente, qui peut être maximum ou minimum selon le glide considéré.
} 